junto al citado manantial salutifero de Cela, un bosque sagrado y celebraba juegos circenses, según la citada inscripción, aunque su nombre no figura en ninguna fuente latina. La primera vez que se cita este topónimo es en El Idrisi, leido por Saavedra como Torchela y Tâyula, de donde, al parecer, procede el nombre actual de Tijola ${ }^{73}$.

R. Láaro Péres: Inseripclones romanas de Almeria. citado, págs. 10-20, A. Tovar: Iberische Landeskunde. Tarraconensis. Baden-Baden. 1989, paigs. 159-160.

\title{
PRIMER AVANCE SOBRE EL GRAN BRONCE CELTIBÉRICO DE CONTREBIA BELAISCA (BOTORRITA, ZARAGOZA)
}

POR

\author{
MARÍA ANTONIA DÍAZ SANZ
}

Directora de la excavación arqueológica

Y

\section{MANUEL M. MEDRANO MARQUÉS}

Departamento de Arqueologia de la Universidad de Zaragoza

\section{RESUMEN}

Se presenta en este trabajo una breve descripción arqueológica y cronológica del yacimiento de Contrebia Belaisca, incidiendo especialmente en los datos proporcionados por las campañas más recientes de excavaciones. Se ofrece una visión global del entorno histórico en que se encuadran los tres documentos escritos sobre bronce hallados en la ciudad, aportando nuevos datos arqueológicos sobre el hallazgo del primer texto celtibérico. El objetivo primordial es dar un avance sobre el gran bronce de Botorrita, aparecido en la campaña de excavaciones de 1992, comentando los datos que proporcionan las circunstancias de su descubrimiento y la lectura de algunos signos que se observaban a simple vista, antes de su limpieza.
SUMMARY

This paper consists of a brief archaelogical and chronological description of the site-find of Contrebia Belaisca, with special emphasis on the data obtained from the most recent excavations. A general view of the historical background in which the documents written on bronze found in the city is given, and new archaelogical data about the finding of the first Celtiberian text are offered. The principal objective is to make a preliminary study of the large bronze found in Botorrita during excavations in 1992, commenting on the data which its discovery provided and the reading of some signs which were seen prior to its cleaning. 
El yacimiento de Comtrehia Belaisca (Botorrita), se halla a unos $20 \mathrm{~km}$ al sur de Zarago/a. junto al rio Huerva. Por lo conocido hasta ahora, la ciudad debio tener una extension de unas $20 \mathrm{H}^{*}$. y ocupaba toda la $/$ ona oriental del actual término municipal de Botorrita (los vestigios comienzan a unos $800 \mathrm{~m}$. al noreste del casco urbano de la localidad) teniendo como zona más elevada el denominado Cabero de las Minas.

Basándonos en los datos obtenidos a través de las campañas arqueológicas efectuadas entre 1980 y 1992 constatamos que en el yacimiento hay una seric de estructuras bien diferenciadas que corresponden a diversas fechas y momentos históricos, en cuanto a su construcción y momento de apogeo.

Utilizando como guia estas estructuras podemos decir que la cronologia general de Contrebia seria la siguiente: los primeros restos de la ciudad corresponden al comienzo del siglo iv a. C.. constatado en el C abezo por la aparición, en la campaña arqueológica de 1992, de un lienzo continuo de muralla de $44.50 \mathrm{~m}$. de longitud, con una orientación este-oeste (figura. 1). Presenta una inflexión acodada de unos $80^{\circ}$ y unos $8.50 \mathrm{~m}$ de longitud en su zona oeste. y una esquina de $90^{\circ}$ con el ángulo abatido en el este, formando un lienzo de $5 \mathrm{~m}$ de longitud hasta encontrarse con otra muralla de adobe, dejando un frente, ligeramente curvado hacia el exterior, de unos $30 \mathrm{~m}$ de longitud. Esta muralla se adaptaria a las lineas generales de

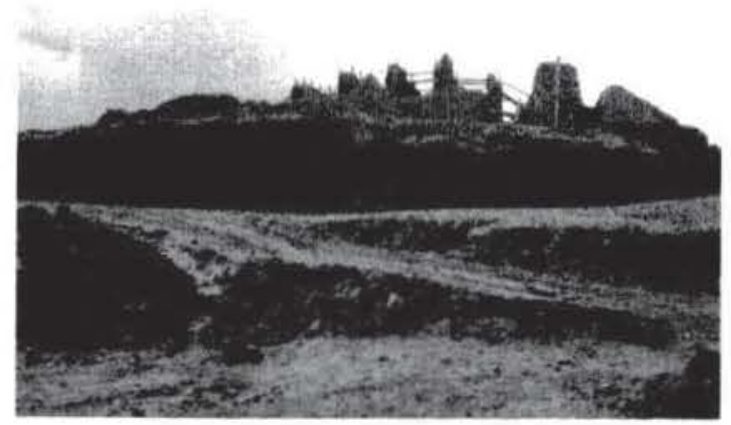

Figura 1. Vista del Cabezo de las Minas desde el su. reste. En primer término, muralla del siglo IV a. C. En la cima, el gran edificio de adobe. la topografia del Cabezo. I a fäbrica es irregular a lo largo de todo el lien/o constituido basicamente por areniscas locales. calizas y. en menor medida. cantos rodados. Los mampuestos estan trabados en seco. hallandose someramente trabajados y calzados con ripio y lajas de arenisca. La mayor altura conservada hasta el momento, ya que no hemos llegado aún a la banqueta fundacional, es de $2.50 \mathrm{~m}$.

En esta muralla aparecieron pocos materiales y de cronologia muy amplia, como cerámica ibcrica con decoración de bandas, dolia tipo Ilduradin y varios fragmentos de cerámica a mano alisada y con decoración a peine. No obstante, la fábrica y forma del lienzo de esta muralla nos remiten, según Maluquer (pảg. 66). a un momento a caballo entre los siglos v y iv a. C

Entre finales del siglo III y comienzos del II a. C. se erigiria el gran edificio de adobe en la zona sur de la cima del Cabezo, aunque abierto y orientado al norte. Su momento de mayor esplendor se sitúa en el siglo $\|$ a. C.. siendo destruido violentamente en la primera mitad del siglo । a. C. (Diaz, M. A., 1987: pág. 230). De este momento de esplendor tenemos también toda una serie de instalaciones fabriles dedicadas al curtido de pieles, que se extienden por la zona noroccidental de la cima del cerro, la ladera norte y este del mismo (Diaz, M. A. y Medrano. M., 1986), y que se encuentran igualmente presentes en el área baja del poblado, en el noreste, cerca del rio (Diaz, M. A., y Torralba, J., pág. 47). El nivel de destrucción de estas tenerias nos proporciona una datación en torno a los años 79-78 a. C., por lo que podemos ponerlo en relación con las guerras sertorianas.

Hacia el 49-48 a. C. (Diaz y Torralba, pág. 43) hay otra fase de violenta destrucción. constatada tanto en el Cabezo (es el momento en que, pese a estar protegido por diversas murallas, es devastado el gran edificio de adobe) como en la zona baja del poblado, donde encontramos este mismo nivel de incendio en la casa agricola excavada en 1970 y donde, en sus estancias de trabajo, apareció el primer bronce escrito de Botorrita.

A partir de este momento, el Cabezo tiene una única funcionalidad: la militar. Se rehacen 
las murallas, a base de recrecimientos y superposiciones de adobe, y las habitaciones que encontramos son pequeños aposentos destinados al cuerpo de guardia de la guarnición.

El final de la ciudad acontece de forma pacifica a lo largo del siglo ॥ d. C., trasladándose a un paraje denominado Monte de San Antonio, al norte del casco urbano de Botorrita (en dirección a Fuendetodos), lugar en el que podria estar enclavada la Trebia que sitúa el $R a-$ vennate por esta zona.

\section{LOS DOCUMENTOS DE BOTORRITA ESCRITOS SOBRE BRONCE}

Con el aparecido en octubre de 1992, son ya tres los bronces escritos hallados en la ciudad de Contrebia Belaisca, dos de ellos en lengua celtibérica, y un tercero en latín.

El bronce latino (tabula Contrebiensis) fue encontrado en una excavación clandestina, y pudo ser finalmente recuperado efectuando su estudio Guillermo Fatás (1980). Al parecer procede de un punto situado en la base del Cabezo de las Minas, prácticamente frente al lugar donde se halló el tercer bronce. Sus dimensiones son $43,8 \times 20,8 \mathrm{~cm}$ y contiene un texto jurídico en el que los jueces del senado contrebiense emiten fallo sobre un litigio entre otras comunidades indígenas, procedimiento judicial que es sancionado apıobatoriamente por el procónsul de la Provincia Hispania citerior. Se data la actuación el 15 de mayo del 87 a. C.

El primer bronce celtibérico se halló en la campaña de excavaciones arqueológicas realizada en 1970. Según los datos proporcionados por el profesor Manuel Martín-Bueno, responsable de los trabajos de campo en esa campaña, apareció en dos fragmentos. El primero, más pequeño, se encontró en el interior de un patio abierto o semicubierto; el segundo estaba a unos tres metros de distancia y en otra habitación contigua a la anterior. El fragmento mayor se situaba sobre los restos de una placa de madera quemada, de forma regular, a la que pudo estar adherido. Toda esta zona pertenece al área de trabajo de una vivienda, y no a su zona noble. Caben serias dudas de que éste fuera el empla- zamiento original del documento; debió estar ubicado en las proximidades del lugar de aparición. Los materiales arqueológicos evidencian una cronologia final en torno a mediados del siglo । a. C., pudiéndose precisar más mediante algunas ánforas, cerámica ibérica, e imitaciones ibicencas de campaniense, que nos llevarian a época cesariana (Diaz y Torralba, pág. 43). Las dimensiones de este bronce son $40,5 \times 9,5 / 10,5 \mathrm{~cm}$ y es el único de los tres que está escrito por las dos caras. En su cara A presenta un texto de 11 líneas y en la cara B una lista en 9 líneas de individuos con su nombre personal más el gentilicio y el nombre del padre en genitivo singular; las referencias correspondientes a cada personaje terminan con la palabra bintis. Sobre este documento se han efectuado interesantes estudios lingüisticos, como el de Javier de Hoz y Luis Michelena (1974), otros que incluyen algunas referencias arqueológicas (A. Beltrán y A. Tovar, 1982) y trabajos más recientes que han intentado profundizar en el análisis filológico o incluso plantear hipótesis acerca del significado de su contenido (J. F. Eska, 1989; G. Olmsted, 1991).

El denominado gran bronce de Botorrita apareció el 20 de octubre de 1992, al efectuar una serie de catas arqueológicas preventivas en el lugar donde se habia previsto situar un aparcamiento de automóviles. Se trataba de unos trabajos secundarios incluidos dentro de la campaña de excavaciones arqueológicas y las obras de cubrición del Cabezo de las Minas, dirigidas por María Antonia Díaz Sanz. El lugar de hallazgo se ubica en una pequeña elevación del terreno al sureste del Cabezo, distando $88,30 \mathrm{~m}$ de la muralla del siglo iv a. C. y unos $65 \mathrm{~m}$ del foso que rodea al Cabezo por el sur (figura 2). Existen noticias antiguas de la presencia de opus tesellatum en el inmediato entorno de este lugar (J. J. Pamplona, pág. 148) y más recientes del hallazgo de glandes de honda de plomo, monedas $y$, en las proximidades, fragmentos cerámicos y restos constructivos, todo ello fuera del área vallada del yacimiento.

Estratigráficamente apareció en un nivel arcilloso, que se asienta sobre otro de arenisca en el que se incrustaba una esquina del bronce. Sobre la capa de arcilla había otra de gravilla 


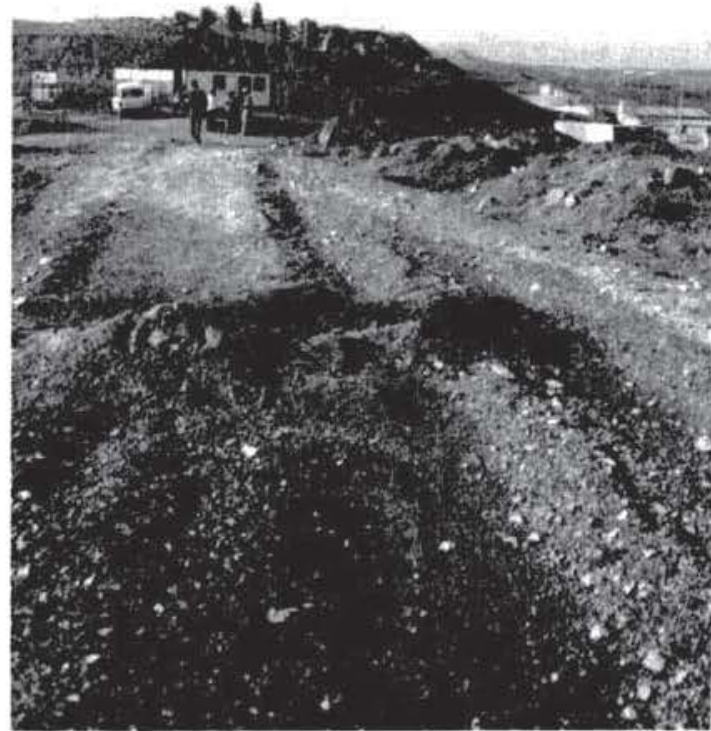

Figura 2- Fn primer termino. lugar de hallasgo del gran bronec de Botorrita. Al fondo. Cabezo de las Minas.

natural que, poco antes de efectuarse la cata, fue parcialmente retirada por los vecinos de Botorrita.

Fisicamente se trata de una placa de $73 \mathrm{~cm}$ en el sentido de la escritura, por $52 \mathrm{~cm}$ y con un grosor que oscila entre 6 y $4 \mathrm{~mm}$. Apareció partida en dos grandes fragmentos, recuperándose después otros 92 fragmentos de dimensiones variables, correspondientes a la esquina superior derecha (figura 3). Los análisis metalográficos indican la presencia de plomo en la aleación, asi como de burbujas en el interior

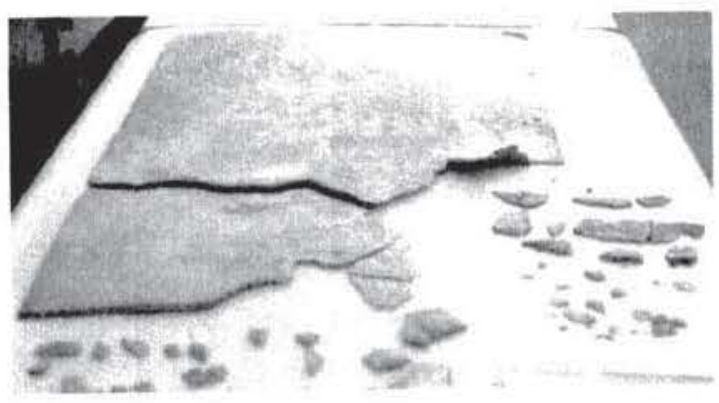

Figura 3.- Vista del gran bronce de Botorrita. del menor de los dos fragmentos mayores. el cual carece de escritura.

Mientras que los dos bronces anteriores mostraban huellas de fuego ( $\sin$ duda como consecuencia de incendios provocados por la destrucción bélica de la ciudad), éste no presentaba alteración termica alguna. Por lo que respecta al sistema de sujecion, mientras que la tahula Contrehiensis posee seis perforaciones regulares e iguales alineadas a intervalos regulares cerca de los dos lados mayores de la placa (Fatas, pág. 15). y el anterior bronce celtiberico tenia quizá un sistema de sustentación a base de tres pequenos orificios por los que se pasarian tal vez los tirantes de sujeción (De Hoz y Michelena, pág. 103). el gran bronce de Botorrita muestra seis orificios circulares, ordenados formando dos triángulos con la base paralela al borde superior de la placa, muy cerca del mismo, y prácticamente en el centro de su longitud. Quedan todavia restos de los clavos en estos orificios, cuya factura no afectó al texto. al contrario de to que sucede con el orificio superior central de la tabula Comtretiensis. Dado que la escritura se extiende hasta el limite del lado izquierdo y del borde inferior del gran bronce, resulta evidente que se expuso sujeto por los dos grupos de orificios citados que forman triangulos, quedando libre el resto.

\section{EL GRAN BRONCE DE BOTORRITA}

El documento presenta una buena ordinatio en sus lineas que, al igual que en el caso del bronce latino, hace pensar que su preparación fue cuidadosa y que, tal vez, se trazara previamente un borrador o plantilla (Fatas, pág. 16). A diferencia de los otros dos textos, aqui la leyenda está realizada con la técnica del puntillado y, como en el anterior celtibérico, las palabras se separan mediante dos puntos, como puede apreciarse en las lineas n. ${ }^{\circ} 3,5$ y 7 que transcribimos más adelante.

El gran bronce de Botorrita, a simple vista y antes de su limpieza, parece contener unas 50 lineas de texto, en lengua celtibérica y escritas en semialfabeto ibérico, si bien no seria de extrañar la presencia de alguna influencia 
lingüistica del ibero, dada la situación culturalmente fronteriza de Contrebia Belaisca. La extensión del documento le confiere una extraordinaria importancia, puesto que estamos ante el más amplio texto celtibérico conocido. lo cual supone un aporte importante para intentar descifrar esta lengua y, si logramos averiguar su contenido, también para profundizar en el conocimiento de la cultura celtibérica. Por otra parte el hallazgo de tres textos sobre soporte broncineo en Contrehia, permite plantear seriamente la hipótesis de que existiera un archivo en la ciudad que, dada la proximidad de los lugares de aparición de dos de los documentos, debió situarse cerca del punto donde encontramos el gran bronce.

Aunque en el momento de escribir estas lineas la única actuación llevada a cabo es la limpieza de la tierra adherida a la placa metálica, pueden apreciarse ya fragmentos de texto en algunas partes de su superficie. A modo de ejemplo transcribimos a continuación algunos signos que se aprecian a simple vista en nueve lineas situadas bajo los clavos de sujeción, que ya podian leerse cuando se extrajo la pieza:

$$
\begin{aligned}
& 1 \ldots A N N \uparrow \ldots \uparrow \ldots \\
& 2 \ldots M \otimes[-] N \ldots M I \ldots \\
& 3 \ldots \times[-] \otimes t: \leqslant N \Psi M \otimes t \backsim \sim \vdash \wedge \Psi Y[-] \leq \| M . \\
& 4 \ldots 4[-] N \angle M H M \uparrow N \triangleright N P M \odot \Psi \ldots \\
& 5 \ldots \wedge \wedge[-] N \vee M: \uparrow \wedge \uparrow \kappa M \diamond \uparrow \ldots \\
& 6 \ldots \uparrow \wedge \uparrow \uparrow N N \diamond \uparrow \ldots
\end{aligned}
$$

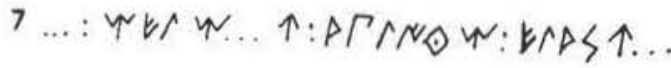

$$
\begin{aligned}
& 8 \ldots \diamond \uparrow \ldots \\
& 9 \ldots N M \ldots
\end{aligned}
$$

La transcripción en alfabeto latino de estos fragmentos textuales seria:

1. ...cainu...m...

2. ...śde(-)n...śba...
3. ...tat - )re: centiścuemelmy(-)sbabaś...

4. ...ti(-)neśosuiriaścum...

5. ...cal(-)ies: mlriścum...

6. ...ucarunicum...

7. .... melm...u: abilicum: elasu...

8. ...cum...

9. ...lis...

Aunque esto no es más que una muy breve muestra del contenido del bronce $y$, desde luego, el primer análisis del texto deberá ser de indole filológica, materia en la que no somos especialistas, podemos ya realizar algunos comentarios:

En la línea n. ${ }^{\circ} 3$, observamos la presencia de la conjunción enclítica -cue, conocida en otras inscripciones celtibéricas y que también aparece en el anterior bronce de Botorrita (cara A; v. Beltrán y Tovar, pág. 37). En las lineas n. ${ }^{\circ} 3$ y 7 encontramos el antropónimo melmu, que se repite tres veces en la cara B del bronce precedente (op. cit., pág. 37). En la linea n." 3 aparece el signo " $Y$ », del cual ya tenemos otros testimonios epigráficos en Contrehia Belaisca y que, como ya expresamos en un trabajo anterior, debe utilizarse en esta zona como vocal (M. Medrano y M. A. Diaz, 1986). En la línea n. ${ }^{\circ} 7$ observamos la palabra elusu, que cabe poner en relación con la cita que efectúa A. Beltrán (op. cit., pág. 57) de una inscripción sobre una pieza de alabastro, procedente de Contrehia, que contiene la leyenda Munip. Elaisio, y que podria hacer referencia a una entidad municipal.

Finalmente por lo que respecta a la cronologia del gran bronce lo único que podemos decir, mientras no avance el trabajo arqueológico en la zona de hallazgo, es que debe ser coetáneo del anterior bronce celtibérico y que, por los materiales aparecidos en el inmediato entorno (los glandes de honda citados), su abandono debe coincidir con algún acontecimiento bélico.

\section{BIBLIOGRAFIAA}

Beltrán, A y Tovar, A. (1982): CONTREBIA BELAISCA (Botorrita, Zaragoza), I, El bron- 
ce con alfabeto "ibèrico" de Botorrita, $\mathrm{Za}$ ragoza.

DI: Ho/, J., y MICHLLL:NA, L. (1974): La inscripción celtibérica de Botorrita, Salamanca.

DiAz SANZ, M. A. (1987): Evolución estructural y funcional en el «Cabezo de las Minas» de Botorrita (Zaragoza): siglos ı a. C. a । d. C., I Jornades Internacionals d'Arqueología Romana, 230-235, Museu de Granollers.

Diaz. Sanz, M. A., y Medrano Marquis, M. (1986): Las áreas fabriles de Contrebia Belaisca (Botorrita, Zaragoza): una unidad de producción, Arqueologia Espacial 9, Coloquio sobre el Microespacio 3, 187-207, Teruel.

Diaz Sanz, M. A., y Torralba Martin, J. (1989. 1990): Las ánforas de Contrebia Belaisca: precisiones cronológicas, Caesaraugusta 6667, 29-55, Zaragoza.

Esk^, J. F. (1989): Towards an interpretation of the hispano-celtic inscription of Botorrita, Innsbruck.

FATAS, G. (1980): CONTREBIA BELAISCA (Botorrita. Zaragoza), II, Tabula Contrebiensis, Zaragoza.

Maluquir de Montes, J., et alii (1986): Arquitectura i urhanisme ibèrics a Catalunva, Universidad de Barcelona.

Midirano, M., y Diaz. Sanz, M. A. (1986): Inscripción ibérica sobre vasija tipo «llduradin» hallada en Contrebia Belaisca (Botorrita, Zaragoza), Estudios en Homenaje al Dr. Antonio Beltrán Martinez, 601-611, Zaragoza.

Olmsted, G. (1991): Gaulish, Celtiberian and Indo-European Verse. The Journal of IndoEuropean Studies, Volume 19, n. ${ }^{\circ} 3$ y 4 , 259-307, Virginia.

PAmplona, J. J. (1957): Breve nota de un yacimiento inédito en Botorrita. Caesaraugusta 9-10, 147-150, Zaragoza.

\title{
TIPOS CERÁMICOS Y GEOQUÍMICA: EL CASTRELÍN DE SAN JUAN DE PALUEZAS (LEÓN).
}

POR

\author{
V. GALVÁN*, M. D. FERNÁNDEZ-POSSE**, \\ F.-J. SÁNCHEZ-PALENCIA*** Y J. GALVÁN* \\ ${ }^{*}$ Centro de Ciencias Medioambientales. CSIC. ${ }^{* *}$ ICRBC. \\ Ministerio de Cultura. *** Centro de Estudios Históricos. CSIC
}

RESUMEN

Se discuten algunos métodos de caracterización mineralógica de cerámicas, buscando el establecimiento de tipos que permitan combinar los datos morfométri$\cos$ y la funcionalidad de las vasijas con su estructura fisico-química. Para ello se utilizan varias categorías cerámicas, análisis de fluorescencia de rayos $\mathrm{X}$ y un tratamiento de los datos con análisis multivariante de los óxidos mayoritarios y minoritarios en la composición de la cerámica. Los materiales analizados proceden de castros prerromanos de la Zona Arqueológica de Las Médulas, caracterizados por unas producciones a mano que se engloban dentro de la Cultura Castreña del Noroeste.
SUMMARY

It is discussed some methods of mineralogic characterization of pottery in order to establish a typology that combines morphometric data and the functional character of pottery with its chemical and physical structure. There are used some ceramic groups treated with fluorescence X-rays in which oxides have been analysed. The material used, typical of the Cultura Castre$\tilde{n} a$, is all handmade and come from prerroman sites of the Zona Arqueológica de Las Médulas. 